\title{
An interactive data-generating and answer-correcting system for problems in statistics
}

\author{
DRAKE R. BRADLEY \\ Bates College, Lewiston, Maine 04240
}

\begin{abstract}
A computer-assisted instructional system is described which provides the student with extensive drill and feedback in the application of statistical methods to computer-simulated data. The system consists of three interactive programs that perform the following functions: (1) FILEMAKE creates "source" data for problems in statistics having whatever population characteristics are desired by the instructor; (2) DATASET generates unique data for each student by performing a random modification, based on sampling theory, of the source data; and (3) CORRECT corrects each student's unique answers to the problem.
\end{abstract}

A problem faced by instructors of introductory statistics is the selection of problems for illustrating the various statistical methods taught in the course. Homework problems provided in most textbooks are generally too few, too limited in scope, or too unrealistic to be adequate for this purpose, and the instructor has no input in determining the content of the problem. The instructor can, of course, design tailor-made problems for illustrating the various statistical techniques, distributing these in the form of handouts. However, this requires the instructor to manually construct and analyze the data for each such problem. Furthermore, since the instructor is usually interested in illustrating some psychological principle with each problem, manual construction of the data is complicated by the requirement that the data, across conditions, display the appropriate overall pattern of results. Some tedious trial-and-error activity is required to construct data having the means and standard deviations arrayed properly across conditions, while simultaneously insuring that the analysis of the data will generate a realistic result in terms of a test statistic. This problem is particularly acute if the instructor wishes to manually construct data having some specific degree of correlation between conditions, as in regression, correlation, or correlated t-test applications. Finally, even if the instructor goes to this effort, the students will always be analyzing the same data, and for this reason may be tempted not to do their own work. At the very least,

The interactive system described in this paper is currently operating on the Dartmouth Timesharing System (DTSS) and is publically available in a statistical sublibrary commanded by the author. Requests for reprints and/or details of the sublibrary should be addressed to Drake R. Bradley, Bates College, Department of Psychology, Lewiston, Maine 04240. The author thanks Susan Dumais, Charles Hotchkiss, Sandra Shea, and Steve Cutcomb for assistance in developing and testing an earlier prototype of the interactive system reported in this paper. such an arrangement encourages students to be more concerned with comparing their answers with each other than with learning the useful skill of crosschecking their own analyses internally,

The data-generating and answer-correcting system described in this paper circumvents the problems mentioned above by using a software package consisting of three interactive programs. FILEMAKE permits problems to be easily set up with the appropriate pattern of results in the data file, without requiring the instructor to manually construct data. DATASET generates different sets of data for each student for any particular problem, so that each student must independently analyze, cross-check, and interpret his or her own results. Finally, CORRECT provides appropriate feedback for each student's individually unique analysis of the same problem.

To illustrate these programs, we consider a problem based on research conducted by Bergin (1962), who investigated the effects of communicator credibility (high vs. low) and communication discrepancy (moderate, high, and extreme) on the amount of attitude change. A general description of this research is prepared and distributed to the students as the first stage of a homework assignment. (Subsequent stages involve the acquisition and analysis of computersimulated data, and verification of the answers obtained.) The description is sufficiently complete to allow the student to deduce the independent variables, the dependent variable, the experimental design, and the appropriate statistical analysis for analyzing the data. In Bergin's study, the dependent variable is change in attitude, as measured by the difference between the subjects' pre- and postcommunication ratings on a 13-point scale (positive change indicates a shift in attitude in the direction endorsed by the communicator).

Table $1 \mathrm{~A}$ presents the mean and standard deviation 
of the change scores for each experimental condition as reported by Bergin (1962, p. 432). Table 2A presents the analysis of variance reported for this data (p. 431), and Figure $3 \mathrm{~A}$ shows the mean change in attitude as a function of communication discrepancy and communicator credibility (p. 433). With regard to the computer-assisted instructional (CAI) system to be discussed, the task at hand is threefold. First, we must find some way of allowing the instructor to represent the information in Table 1A as "source" data in the computer, from which data can be generated individually for each student. Since raw data are not provided in the research report, this means that a reasonable facsimile of that data must be constructed from the information in Table $1 \mathrm{~A}$. Second, we must find a method for generating data from the source data that guarantees that each student will receive a unique set of data, but such that this data can later be reconstructed (from an appropriate code) for the purpose of correcting the student's answers. Furthermore, in the interest of realism and proper statistical technique, it would be very desirable if the variation in the data sets generated across students could simulate that which would be expected on the basis of sampling theory. Third, once the student has analyzed the data, it is important that immediate feedback be available concerning the correctness of the answers obtained. We consider next the computer programs written to achieve these goals.

\section{PROGRAM DESCRIPTIONS}

The three interactive programs and the data files created by the instructor require about $15-20 \mathrm{~K}$ of storage. The programs are written in BASIC and are self-contained with the following exception: Subprograms that return $z, t$, and $F$ probabilities, and one that returns a random standard normal deviate (z), are all called from an external statistical library. These subprograms can be easily incorporated into the calling programs if they are not available on other computer systems. The three interactive programs will be considered in turn.

\section{FILEMAKE}

This interactive program allows the instructor to compose normally distributed data having whatever characteristics are appropriate for a particular problem. The data can be discrete or continuous, and if continuous, the program can retain as many places to the right of the decimal place as appropriate for the type of variable being measured. In running the program, the instructor first specifies the output file name for the data file, and then the primary analysis to be performed on the data. Examples of primary analyses handled by the system are: one-sample $t$, independent $t$, correlated $t$, regression, correlation, correlation 2 (for testing differences between correlations), ANOVA, and ANOVA 2 (for two-way analyses of variance). Depending on the primary analysis specified, the program then requests whatever information is necessary to compose data for that type of problem. Group labels, sample sizes, means, and standard deviations are requested for each condition, for all problem types. If the problem involves correlation, regression, or correlated observations, the program requests the true correlation between conditions as well. Finally, if the problem involves a one-sample test, the theoretical value of the population parameter under the null

Table 1A

Means and Standard Deviations for Change Scores (Bergin, 1962)

\begin{tabular}{|c|c|c|c|c|c|c|}
\hline & \multicolumn{6}{|c|}{ Credibility Condition } \\
\hline & \multicolumn{3}{|c|}{ High } & \multicolumn{3}{|c|}{ Low } \\
\hline & \multicolumn{3}{|c|}{ Discrepancy Condition } & \multicolumn{3}{|c|}{ Discrepancy Condition } \\
\hline & Moderate & High & Extreme & Moderate & High & Extreme \\
\hline Mean & 1.30 & 2.10 & 2.20 & .80 & .40 & .20 \\
\hline $\mathrm{SD}$ & .68 & 1.37 & 1.61 & .92 & 1.26 & .79 \\
\hline \multicolumn{7}{|c|}{$\begin{array}{c}\text { Table 1B } \\
\text { Means and Standard Deviations for Change Scores } \\
\text { (CHANGE data file) }\end{array}$} \\
\hline & \multicolumn{6}{|c|}{ Credibility Condition } \\
\hline & \multicolumn{3}{|c|}{ High } & \multicolumn{3}{|c|}{ Low } \\
\hline & \multicolumn{3}{|c|}{ Discrepancy Condition } & \multicolumn{3}{|c|}{ Discrepancy Condition } \\
\hline & Moderate & High & Extreme & Moderate & High & Extreme \\
\hline Mean & 1.30 & 2.10 & 2.20 & .80 & .40 & .20 \\
\hline SD & .68 & 1.36 & 1.62 & .92 & 1.26 & .77 \\
\hline
\end{tabular}

Note $-N=10$ for each condition.

Table 2A

Analysis of Variance of Change Scores (Bergin, 1962)

\begin{tabular}{lrrc}
\multicolumn{1}{c}{ Source } & df & \multicolumn{1}{c}{ MS } & \multicolumn{1}{c}{ F } \\
\hline Credibility (A) & 1 & 29.40 & $21.99^{*}$ \\
Discrepancy (B) & 2 & .22 & .17 \\
Interaction (A by B) & 2 & 3.14 & $2.35^{* *}$ \\
Error (within cell) & 54 & 1.34 & \\
Total & 59 & & \\
\hline
\end{tabular}

Table 2B

Analy sis of Variance of Change Scores (CHANGE data file)

\begin{tabular}{lrrc}
\hline \multicolumn{1}{c}{ Source } & df & \multicolumn{1}{c}{ MS } & \multicolumn{1}{c}{ F } \\
\hline Credibility (A) & 1 & 29.40 & $22.14^{*}$ \\
Discrepancy (B) & 2 & .22 & .16 \\
Interaction (A by B) & 2 & 3.15 & 2.37 \\
Error (within cell) & 54 & 1.33 & \\
Total & 59 & & \\
\hline
\end{tabular}

$* p<.001$

**In the original table, this value was computed incorrectly $(F=2.48)$. 
hypothesis is requested. The program writes the data and other necessary information into the data file according to a format standardized for each problem type.

Figure 1 shows a sample interactive sequence of the instructor using FILEMAKE to compose data for a problem based on the Bergin (1962) study, followed in turn by a listing of the data file (CHANGE). Note that the group labels, sample sizes, means, and standard deviations input to the program are simply those reported by Bergin (Table 1A). The data composed by FILEMAKE for each condition, as seen in the listing, are normally distirbuted with mean, standard deviation, and accuracy (.1 of a scale unit) as requested by the instructor. The means and standard deviations of the composed data are presented in Table 1B for comparison with the original. An analysis of variance of this composed data is presented in Table $2 \mathrm{~B}$, again for comparison with the original (Table 2A). FILEMAKE has successfully produced data embodying the essential characteristics of the original (unreported) raw data with regard to central tendency, variability, and the overall pattern of results (ANOVA).
FILEMAKE composes normally distributed data in the following manner. For each condition, a cumulative probability space ranging from 0 to 1 is divided into $\mathrm{N}$ equal intervals, and the midpoint of each interval is used to represent the cumulative probability of a standard normal deviate. The corresponding $z$ value for each such probability is obtained using standard approximation techniques (Zellen \& Severo, 1965). The resulting $z$ values are then converted to $X$ values, with mean and standard deviation as specified by the instructor, and written into the data file in a random order. Problems requiring correlation between conditions are treated somewhat differently. The program first obtains $\mathrm{N}$ standard normal deviates, as just described. A constant is then added and subtracted to each $z$ value to obtain pairs of values arranged as follows: $z_{1}+c, z_{1}-c ; z_{2}-c, z_{2}+c$; and so on. If the constant is selected so that $c=\sqrt{(1-r) /(1+r)}$, where $\mathrm{r}$ is the desired degree of correlation requested by the instructor, then the two "adjusted" sets of $\mathrm{z}$ scores are correlated by about this amount. (This is an oversimplification of the actual procedure used, but will suffice for the present purpose.) FILEMAKE

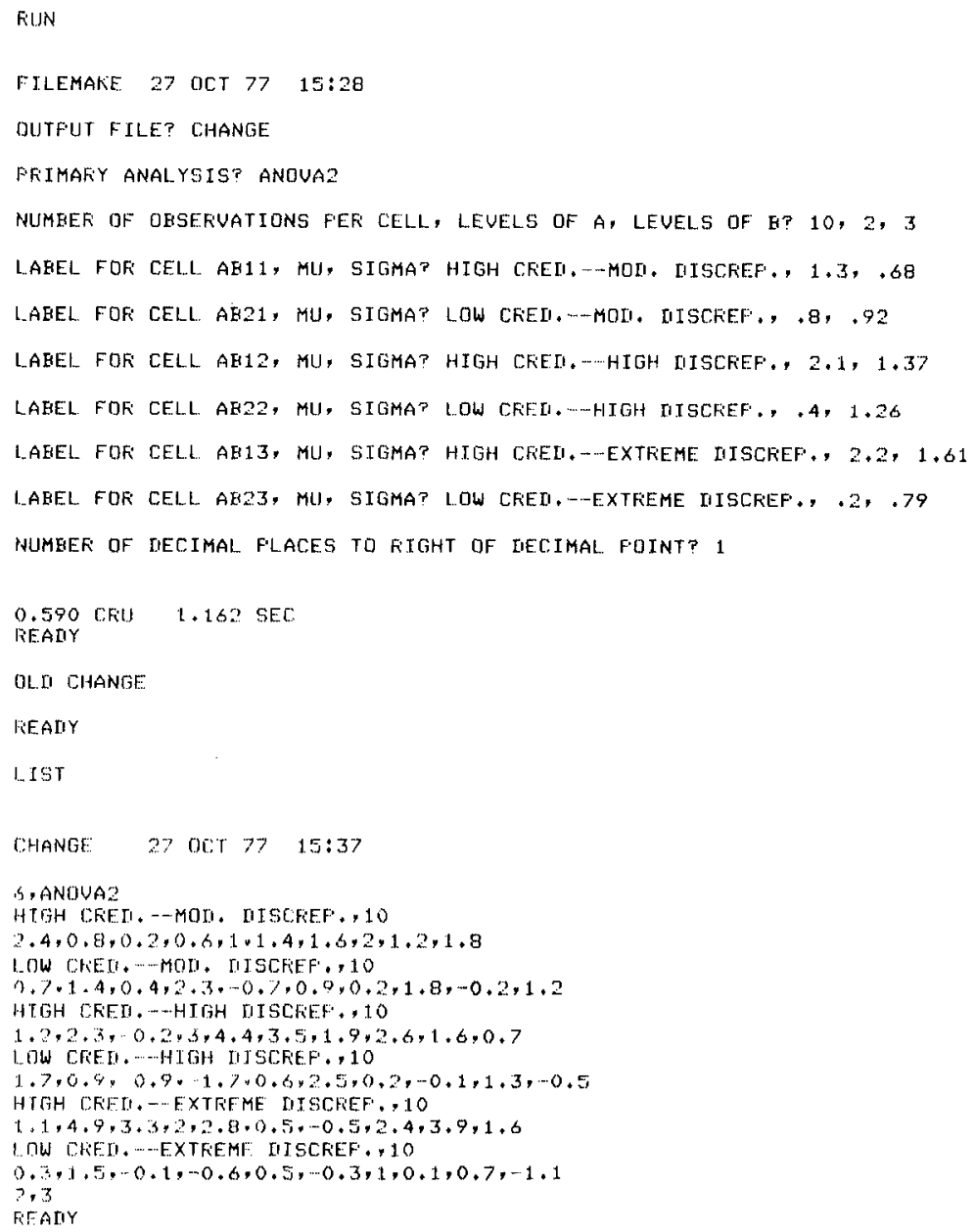

Figure 1. An interactive sequence of the instructor using FILEMAKE to create data for the problem CHANGE, followed by a listing of the data file. 
then converts these sets of $\mathrm{z}$ values to $\mathrm{X}$ and $\mathrm{Y}$ data having means and standard deviations as requested and writes the data into the data file in a random but identical order for the two conditions (see below, Figure 6).

As is evident from Figure 1, FILEMAKE allows the instructor to easily compose data for relatively complex problems. Of course, it is not always the case that the instructor will want the data to be normally distributed: Reaction time data, for example, typically display a positive skew. In this case the instructor can compose the source data manually so as to have the appropriate nonnormal form. The data and the other information required can then be manually stored in a data file using the appropriate format for the problem.

\section{DATASET}

This interactive program is used by the student to obtain data for the problem. The problem is described in a handout distributed by the instructor, and the name of the data file containing the "source" data for that problem is indicated at the top of the handout. In running DATASET, the student simply provides the name of the data file. The program accesses the data file, inputs the data for each condition, and modifies the data using a special randomization procedure. The program then outputs the modified data, labeled by group, followed by a set of randomization parameters. The latter are required by the correcting program in order to reconstruct the student's data from the data file.

Figure 2 shows a sample interactive sequence of a student using DATASET to obtain data for the problem CHANGE. The means computed on this modified data are plotted in Figure $3 \mathrm{~b}$ for comparison with the original data reported by Bergin (Figure 3A). Figures 3C-3F show the means of the data obtained from DATASET when four additional students independently "replicate" Bergin's research. The variation in the pattern of means observed across replications simulates that which would arise from sampling error.

Specifically, the randomization procedure employed insures that the following will be true, in the long run, across all sets of data generated for a particular condition: (1) The mean of the means and the mean of the standard deviations across sets will be equal to the mean and standard deviation of the source data in the corresponding condition of the data file; and (2) the standard deviation of the means and that of the standard deviations across sets will be equal to the standard errors of these statistics as computed from the standard deviation and sample size of the source data. (Both of these statements have been verified by empirical simulation.) The source data for each condition define a "population" from which the student's data are sampled by DATASET, and the instructor, in running FILEMAKE to create the source data, is implicitly

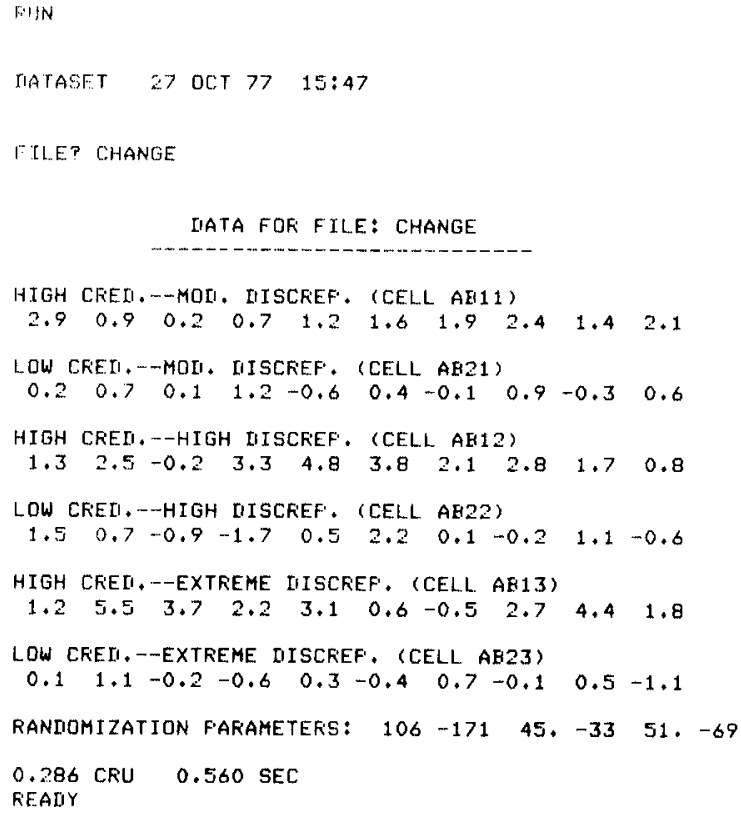

Figure 2. An interactive sequence of the student using DATASET to obtain computer-simulated data for the problem CHANGE.

defining the population parameters $(\mu, \sigma)$ of each condition.

The modification of the source data is achieved by sampling a standard normal deviate at random, and then transforming each $\mathrm{X}$ value by the following rule:

$$
\begin{aligned}
& X^{\prime}=\frac{\sigma+z \frac{\sigma}{\sqrt{2 N}}}{\sigma}(X-\mu)+\left(\mu+z \frac{\sigma}{\sqrt{N}}\right)= \\
& X^{\prime}=\frac{\sigma+z \sigma_{s}}{\sigma}(X-\mu)+\left(\mu+z \sigma_{\bar{X}}\right)= \\
& X^{\prime}=\frac{s^{\prime}}{\sigma}(X-\mu)+\bar{X}^{\prime}
\end{aligned}
$$

The Greek symbols (Equations 1-3) refer to the parameters defining the source data, whereas the primed Latin symbols (Equation 3) refer to the corresponding statistics of the data as modified by the transformation. Note that this procedure "yokes" the random modification of the mean and standard deviation by using the same randomly sampled $z$ score to alter each. In order to independently modify the central tendency and the variability of the data in the data file, two different $z$ scores can be randomly sampled and used in each part of Equation 2. However, this requires that the student specify two randomization parameters, rather than one, for each condition when running the correcting program (see below). Since some ANOVA and ANOVA 2 problems may have nine or more experimental conditions. this requires that 18 or more 

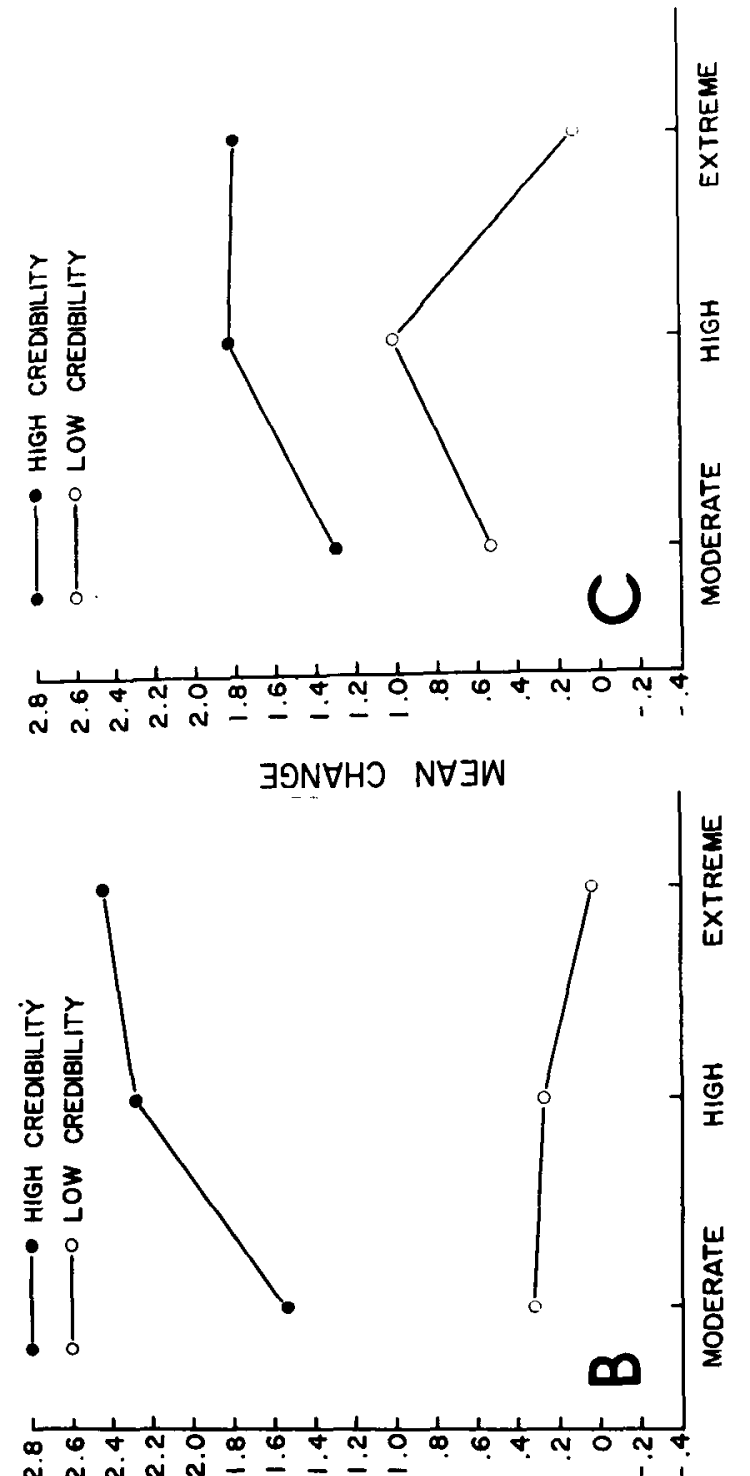

a n a n a n

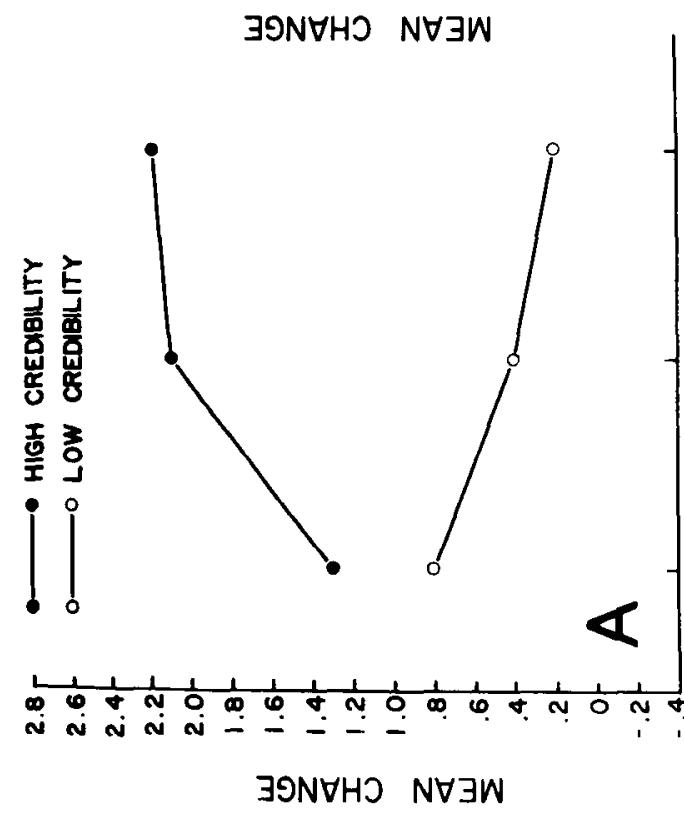

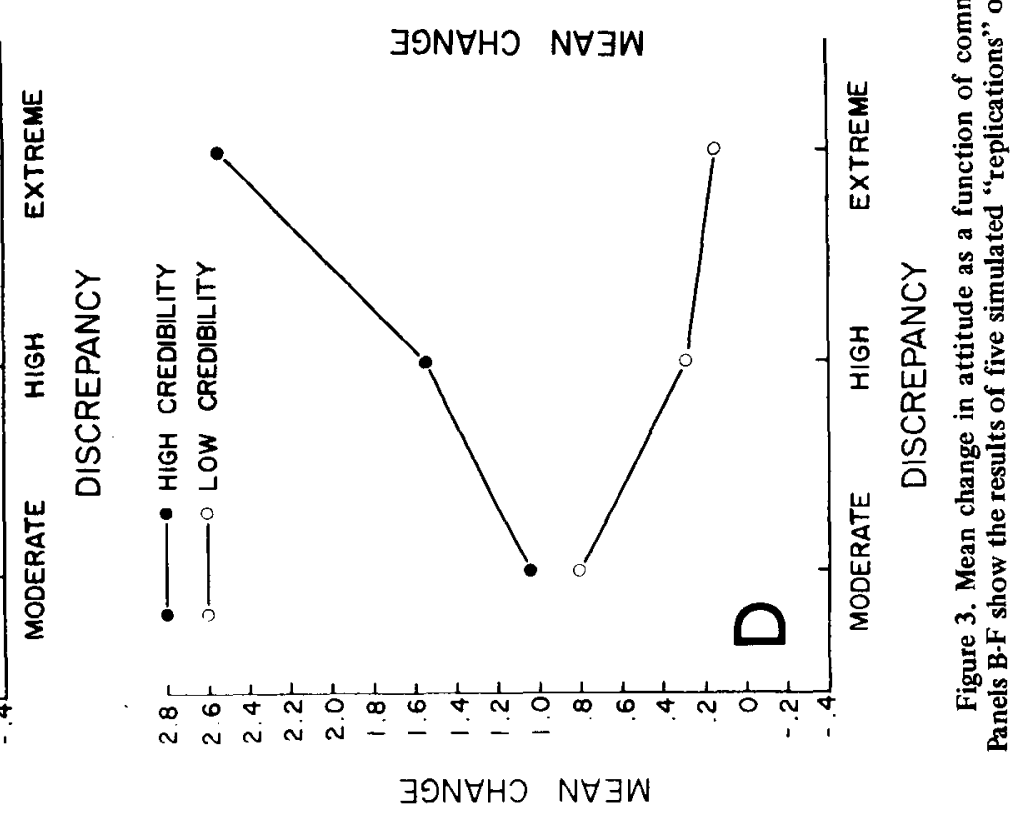


randomization parameters be input to the correcting program before it can reconstruct the data. Consequently, we decided that yoking the two modifications would be necessary for the convenience of the student, even though it represents a concession to maintaining complete realism in the sampling behavior of the mean and standard deviation. (A second concession results from basing the random modification of the standard deviation on a randomly sampled $\mathrm{z}$ score, since this causes its sampling distribution to be normal in form.) Nevertheless, the randomization procedure used by DATASET does guarantee that the mean and standard error of $\bar{X}^{\prime}$ and $s^{\prime}$ across data sets fulfill the requirements stated in the preceding paragraph.

The randomization parameters output by DATASET are simply the randomly sampled $\mathrm{z}$ scores multiplied by 100 . By noting the sign and magnitude of these "coded" parameters, the instructor can immediately determine the direction and size of the modification of the mean and standard deviation of each condition in the data file. In Figure 2, for instance, the first

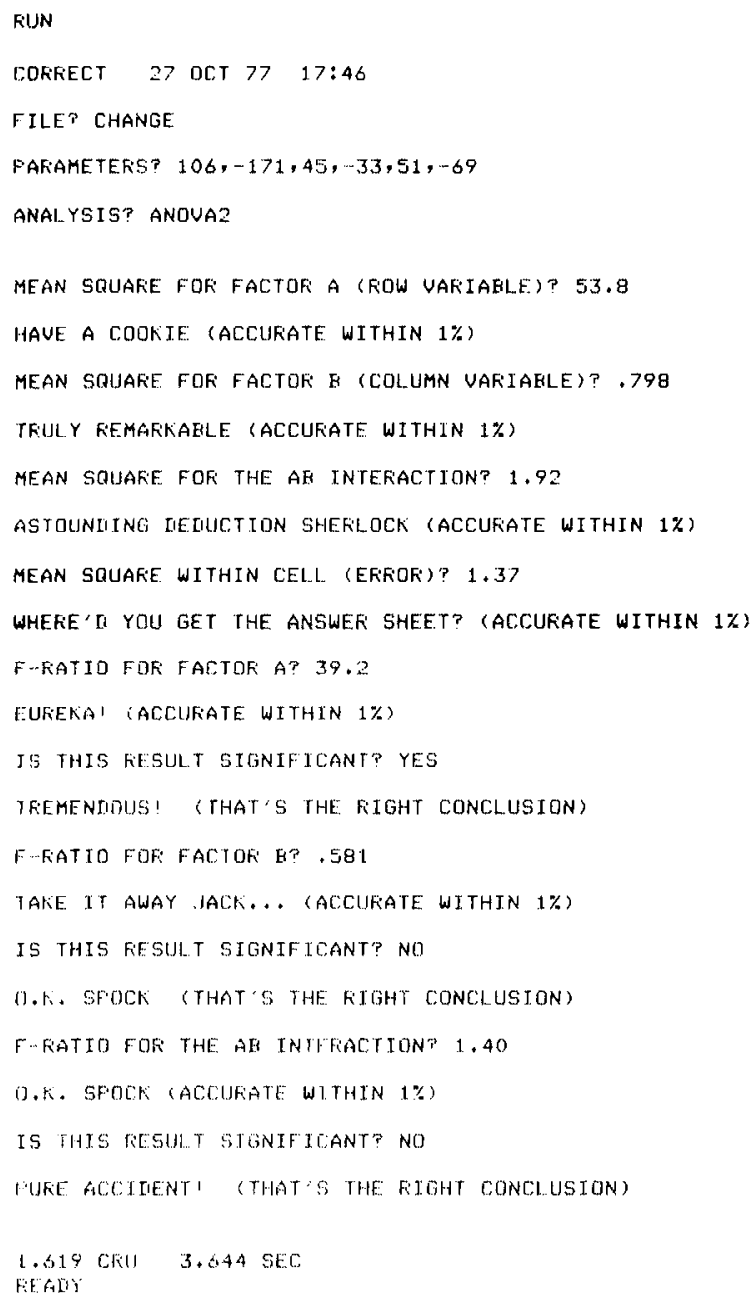

Figure 4. An interactive sequence of a student using CORRECT to verify the answers for a two-way analysis of variance of the data output by DATASET (Figure 2).

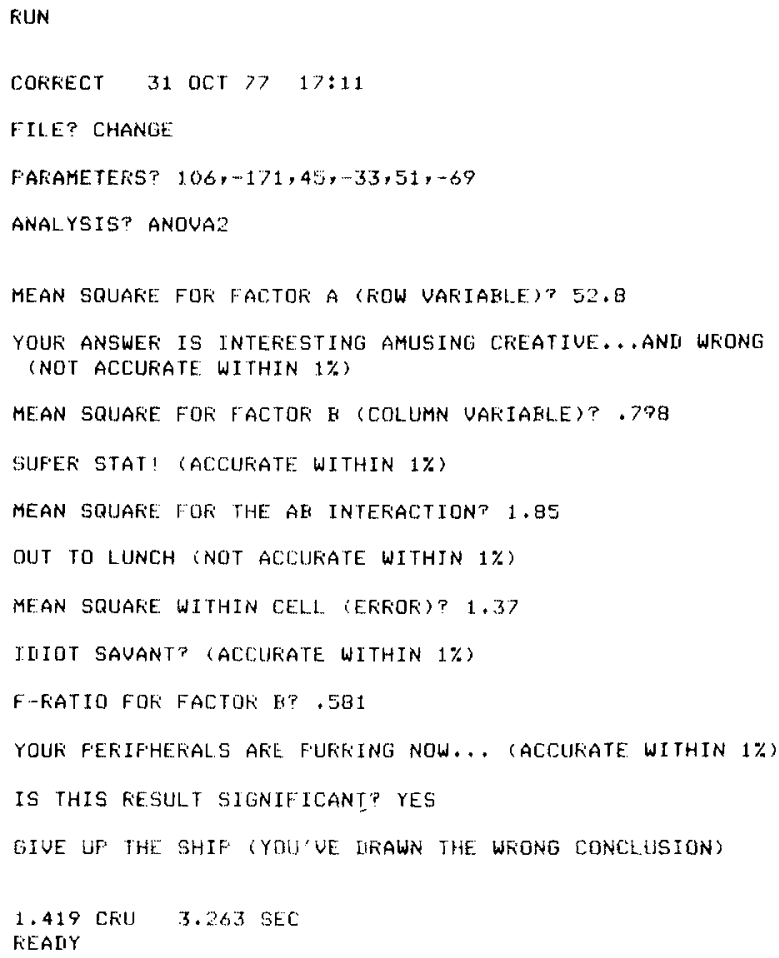

Figure 5. An interactive sequence of CORRECT responding to incorrect values and conclusions submitted by the student.

randomization parameter (106) indicates that the mean of the first group of data has been increased by 1.06 standard errors $\left(\sigma_{\overline{\mathrm{x}}}\right)$ relative to the mean $(\mu)$ of the source data. The students are not, of course, aware of the significance of the randomization parameters. They know only that the correct entry of these numbers to the correcting program is required in order to obtain valid feedback concerning the accuracy of their answers.

\section{CORRECT}

This interactive program is used by the student to determine whether the proper analysis has been performed on the data, and presuming it has, to determine the accuracy of the specific values computed. In addition to correcting any of the primary analyses handled by the system (see above), the program can correct a number of secondary analyses that can be performed on all or some of the problems. For example, STATISTICS (for correcting means and standard deviations) and CONFIDENCE LIMITS (about the mean) are secondary analyses that can be performed on all problems. Multiple $t$ analyses can be performed on ANOVA and ANOVA 2 problems, and simple effects analyses can be performed on ANOVA 2 problems whenever the interaction is significant. Taking the primary and secondary analyses together, the correcting program is capable of accommodating most of the parametric statistical analyses taught in an introductory course. 
Figure 4 shows a sample interactive sequence of a student using CORRECT to verify the answers for a two-way analysis of variance of the data output by DATASET in Figure 2. In addition to specifying the file name for the problem, the student must indicate the randomization parameters associated with the data and the type of statistical analysis performed. CORRECT then interrogates the student with respect to the various quantities computed in the analysis and the decisions made on the basis of any inferential tests. A computer demon, GLITCH, is responsible for providing the positive and negative verbal feedback output by CORRECT in response to the student's answers. In Figure 4, the student has succeeded in computing the analysis correctly and in drawing the proper inferences. However, whenever an answer submitted by the student differs from the correct value by more than $1 \%$ (Figure 5), the GLITCH responds with a negative comment ranging in tone from mild censure to searing contempt. Furthermore, CORRECT subsequently avoids asking for any answer that depends, computationally, on a previously submitted incorrect answer (e.g., if the mean square for Factor $A$ is computed incorrectly, the program will not ask for the $\mathrm{F}$ ratio for Factor $\mathrm{A}$ ). In any event, the student must reanalyze the data until the computational error is eliminated, and then attempt once again to get the answers corrected.

The homework assignment is handed in to the instructor only after the student has successfully computed the correct answers, as verified by CORRECT. Unlike the philosophy pervading many programmed instructional systems, in which the commission of errors in mastering the material is to be avoided at all costs (through the proper administration of positive reinforcement to performances progressively approximating the desired result), the present instructional system makes no attempt to insure that

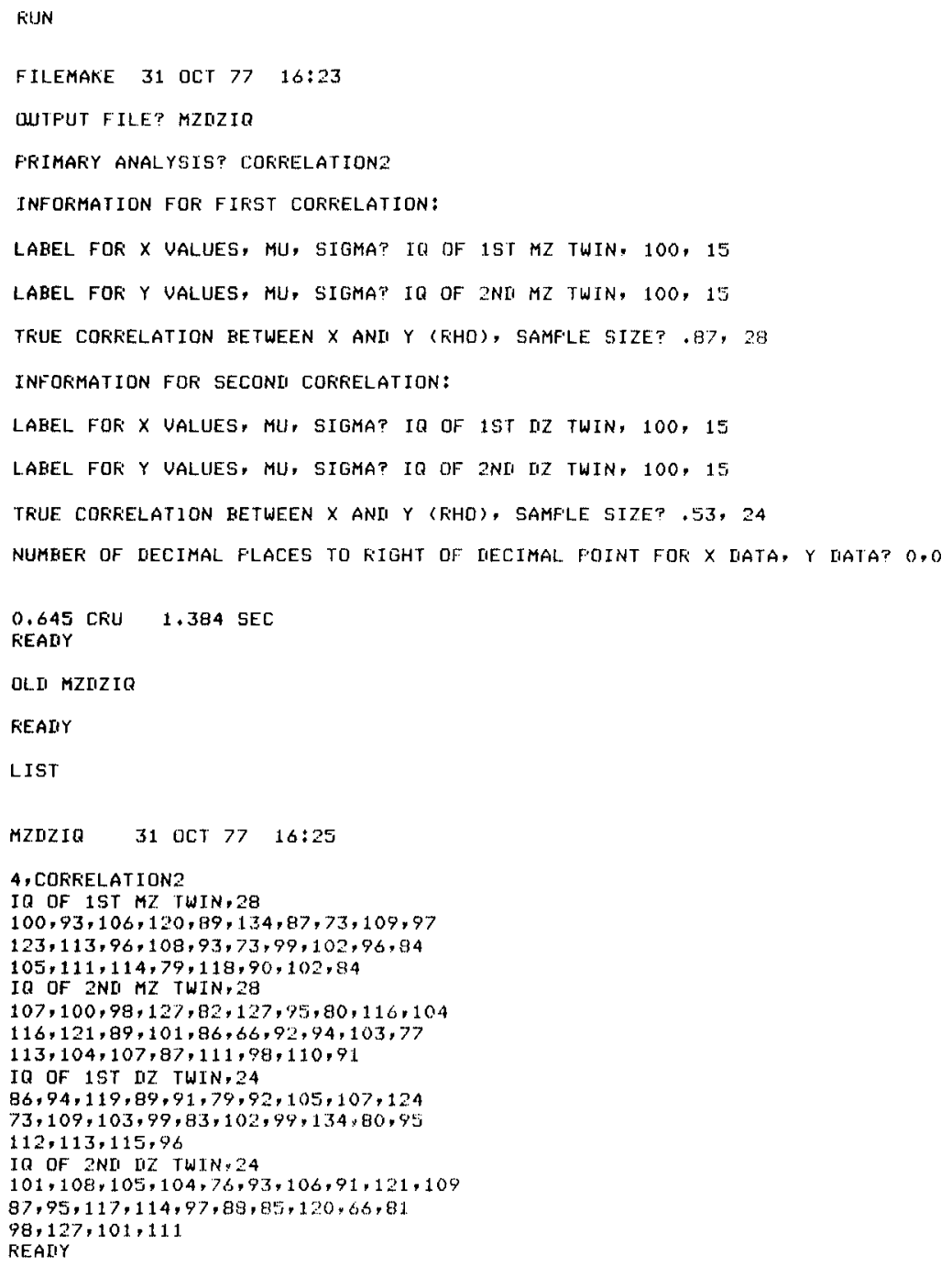

Figure 6. An interactive sequence of the instructor using FILEMAKE to create data for a problem requiring correlational analysis (MZDZIQ), followed by a listing of the data file. 
students avoid making errors. Quite the contrary, an important skill to be acquired by the student is the ability to detect and correct the errors that will inevitably occur when processing large amounts of data in complex statistical analyses.

CORRECT is the largest of the three interactive programs, simply because it must contain statistical routines for performing all of the analyses requiring correction. Consequently, it is structured into four major components. The master program performs the executive functions, such as inputting the file name, randomization parameters, and analysis to be corrected, passing control to the data reconstruction and statistical computing subroutines, and terminating the program under the appropriate circumstances. The data reconstruction subroutine reconstructs the student's unique data by using the (decoded) randomization parameters to transform the source data in the data file in the same manner as DATASET (see above); in addition, this subroutine performs a preliminary analysis of the modified data (means, standard deviations, sums of squares, etc.). The statistical computing subroutines (one-sample $t$, correlated $t$, ANOVA 2, etc.) compute the correct answers for a particular analysis and pass control to the output subprogram to determine the accuracy of the answers submitted by the student. The output subprogram checks the student's answer against the correct value and provides appropriate feedback by accessing (at random) either a positive or a negative verbal comment from an accessory file and printing it out on the terminal.

CORRECT calls subprograms from an external statistical library whenever obtained significance levels are required for $\mathrm{z}, \mathrm{t}$, and $\mathrm{F}$ tests in order to determine whether the test is significant. The program simply establishes whether the returned probability is less than or equal to .05 (except for multiple $t$ tests, in which case the required probability is .05 divided by the number of comparisons) and then interrogates the student as to the decision made (Figure 4).

We conclude this section with a demonstration of the three interactive programs being applied to a problem requiring a correlational analysis (correlation 2 ). The problem is based on data cited by Scarr-Salapatek (1975) in a paper reviewing research on the heritability of intelligence. A frequently encountered paradigm used in this area of research compares the correlation between IQ scores of monozygotic twins to that of dizygotic twins. Scarr-Salapatek reports that, for twins reared together, the median correlation across studies is .87 for monozygotic twins and .53 for dizygotic twins. For the purpose of defining the problem, these correlations are considered to be the true population values $(\rho)$, and the IQ scores are assumed to have a mean $(\mu)$ of 100 and a standard deviation $(\sigma)$ of 15 .

Figure 6 shows the instructor defining the problem MZDZIQ, based on the data reported by Scarr-Salapatek (1975, p. 34). A listing of the data file composed by FILEMAKE is also shown. The correlation between IQ scores for the composed data is .877 for the monozygotic twins and .532 for the dizygotic twins. Hence, FILEMAKE has successfully produced source data having the desired degree of correlation between conditions. Figure 7 shows DATASET generating data from the source data in MZDZIQ, and Figure 8 shows CORRECT verifying the answers for the correlational analysis of the modified data.

\section{CONCLUSIONS}

The data-generating and answer-correcting system reported in this paper allows the instructor to create a wide variety of problems for a course in introductory

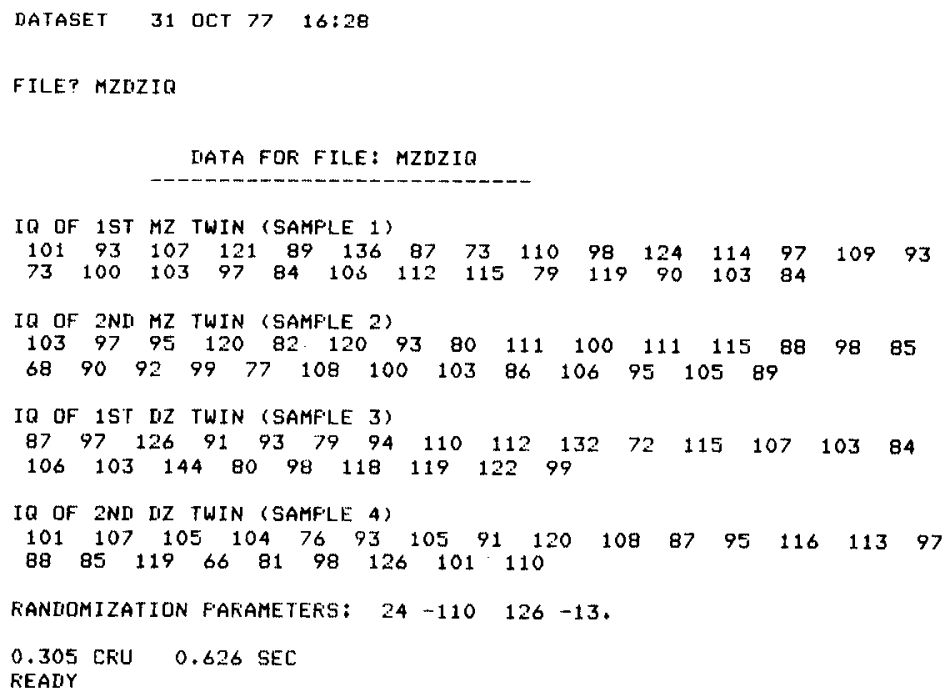

Figure 7. DATASET generating data for the problem MZDZIQ. 


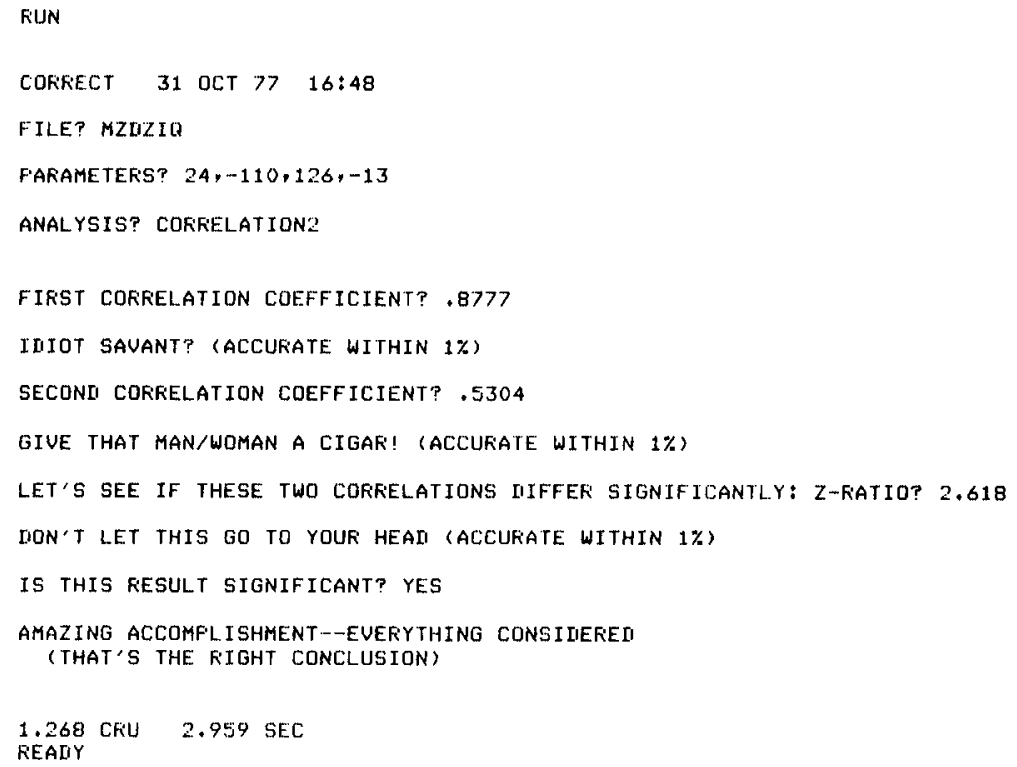

Figure 8. CORRECT verifying the answers to a correlational analy sis of the data output by DATASET in Figure 7.

statistics. Many of these problems can be modeled after the results of actual research conducted in psychology. In this case, statistical analyses of data are illustrated by having the student replicate "classic" experiments or the results of well known contemporary research. Other problems may be created by the instructor based on research scenarios yet to be investigated, but representing logical extensions of contemporary theory and research in a particular area. In this case, the instructor must specify the source data in such a way that the students will be challenged to interpret their data in terms of the theoretical predictions suggested in the handout describing the research. In either case, the instructor need specify only the general characteristics of the data to be created for the problem: central tendency, variability, association (if appropriate), and accuracy. The source data created by FILEMAKE will then define the "population" from which the students' data will be generated.

Short of having students conduct research while enrolled in the course, the data-generating capability of the CAI system reported here comes as close as possible to placing the student in the role of researcher. Indeed, the data generated by the computer can be considered "real" data in the sense that DATASET performs a simulated experiment (or correlational study) that generates data having the same general characteristics as would result from actually doing the research. In particular, by comparing the graphed means of the data generated by DATASET to those of other students (or the original research report, Figure 3), the student can begin to appreciate the effect of sampling error on the outcomes of research. This "replicating" feature of the data-generating system, as based on actual research data, proves to be an extremely valuable teaching heuristic for explaining the principles of sampling theory.

Finally, the individualized correcting capability of the system (CORRECT) allows the instructor to use the time saved in manually correcting assignments for more creative enterprises, such as the construction of Monte Carlo programs for illustrating the various principles of sampling theory. These programs can then be used for computer laboratory assignments associated with the various topics covered in the course (Bradley \& Cutcomb, 1977; Bradley, Hotchkiss, Dumais, \& Shea, 1976; Bradley \& Seely, 1977). The outcome of this approach for teaching statistics is that the computer becomes a general-purpose tool used by the student for three distinct activities: (1) generating computer-simulated data for homework problems and verifying the answers to the statistical analyses conducted; (2) analyzing data using computer programs available in a statistical library, or those written by the student; and (3) conducting Monte Carlo simulations to study the empirical sampling distributions of various statistics.

In summary, the computer-based system described in this paper has five features of special interest to potential users: (1) the system is completely interactive for both the instructor (who must define the problems) and the student (who must solve them), and no expertise in computer programming is required to employ it; (2) the instructor uses an interactive program to define the problem at a high level of generality, usually specifying only the sample size, mean, and standard deviation of each condition, and the program creates normally distributed data fulfilling these requirements and stores them in a data file; (3) the student uses an interactive program to obtain data for the problem 
derived from the "source" data stored in the data file, but randomly modified to some extent so that each student receives unique data requiring individual analysis and interpretation; (4) the variation in the data sets generated across students simulates the actual variation that would occur when a research project is replicated; and (5) the student uses an interactive program to obtain feedback as to whether the analysis performed on the data, the specific values computed, and the inferences drawn are correct.

\section{REFERENCES}

Bradley, D. R., \& Cutcomb, S. Monte Carlo simulations and the chi-square test of independence. Behavior Research
Nethods \& Instrumentation, 1977, 9, 193-201.

Bradley, D. R., Hotchisiss, C. M., Dumais, S. T., \& Shea, S. L. Computer assisted instruction in the small college. Proceedings of the Seventh Conference on Computing in the Undergraduate Curricula, 1976, 205-213.

Bradley, D. R., \& Seely, D. L. Empirical determination of the power of the chi-square test of independence in $2 \times 2$ tables. Proceedings of the Statistical Computing Section of the American Statistical Association, 1977, 138-144.

BERGIN, A. E. The effect of dissonant persuasive communications upon changes in self-referring attitudes. Journal of Personality, $1962,30,423-438$.

SCARR-SALAPATEK, S. Genetics and the development of intelligence. In F. D. Horowitz (Ed.), Review of child development research (Vol. 4). Chicago: University of Chicago Press, 1975.

Zellen, M., \& Severo, N. C. Probability functions. In M. Abramowitz \& I. A. Stegun (Eds.), Handbook of mathematical functions. New York: Dover Publications, 1965. 